\title{
Addresses of reviewers
}

Dr. Dr. Klaus Bergdolt, Centro Tedesco di Studi Veneziani, Palazzo Barbarigo della Terrazza, S. Polo 2765/A, I-30125 Venezia

Dr. Thomas Böni, Lenzstrasse 2, 8340 Hinwil

Prof. Dr. Urs Boschung, Medizinhistorisches Institut, Bühlstrasse 26, Postfach 139, 3000 Bern 9

Prof. Dr. Johann Jakob Burckhardt, Bergheimstrasse 4, 8032 Zürich

Dr. Willem F. Daems, Stollenrain 15, Postfach 306, 4144 Arlesheim

Dr. Maria Luisa Engeler, Flobotstrasse 9, 8044 Zürich

PD Dr. Hansueli F. Etter, Unter-Rüeggental, 8344 Bäretswil

Dr. Urs Leo Gantenbein, Ackeretstrasse 16, 8400 Winterthur

Dr. Beat Glaus, ETH-Bibliothek, Wissenschaftshistorische Sammlungen, Rämistrasse 101, 8092 Zürich

Dr. Christoph Gradmann, Institut für Geschichte der Medizin der Universität, Im Neuenheimer Feld 368, D-69120 Heidelberg 1

Prof.Dr. Felix Gutzwiller, Institut für Sozial- und Präventivmedizin, Universität Zürich, Sumatrastrasse 30, 8006 Zürich

Dr. Michael Hagner, Georg-August-Universität Göttingen, Institut für Geschichte der Medizin, Humboldtallee 11, D-37073 Göttingen

Dr. Aloys Henning, Osteuropa-Institut der Freien Universität Berlin, Abt. Geschichte, Garystrasse 55, D-14195 Berlin 33

Dr. Lucienne Hubler, Cäcilienstrasse 5, 3007 Bern

Dr. Lies Huizink, General-Wille-Strasse 15, 8002 Zürich

Alfred G. Kauertz, Lötschgraben 6, 3904 Naters

Dr. Hans Urs Keller-Schnider, Mühleackerstrasse 539, 4707 Deitingen

Dr. Claus Kiefer, Institut für Theoretische Physik, Universität Zürich, Schönberggasse 9, 8001 Zürich

Dr. Jürg Knessl, Ärztehaus Hirslanden, Witellikerstrasse 40, 8008 Zürich

Prof. Dr. Huldrych M. Koelbing, Gotthardstrasse 65, 8002 Zürich

Micheline Louis-Courvoisier, Institut Louis Jeantet d'histoire de la médecine, Case postale, 1211 Genève 4

Dr. Antonie M. Luyendijk-Elshout, Prins Bernhardlaan 60, NL-2341 KL Oegstgeest

Dr. Roger Mayer, 9, rue Michel-Chauvet, 1208 Genève

Prof. Dr. Christian Müller, Herrengasse 23, 3011 Bern

Prof. Dr. Andreas P. Naef, Avenue de Villardin 12, 1009 Pully

Prof. Dr. Frank Nager, Medizinische Klinik, Kantonsspital, 6000 Luzern 16

Prof. Dr. François Reubi, Chemin du Village 44, 1012 Lausanne

Guy Saudan, historien, Ecureuils 3, 1009 Pully

Dr. Hans Konrad Schmutz, Naturwissenschaftliche Sammlungen, Museumstrasse 52, 8402 Winterthur

PD Dr. Dr. Gottfried Schramm, Am Brunnenbächli 12, 8125 Zollikerberg

Dr. Roger Seiler, Hadlaubstrasse 47, 8006 Zürich

Dr. Guido Sigron, Aathalstrasse 19, 8610 Uster

Prof. Pierre Speziali, 2, chemin de Roches, 1208 Genève 
Dr. Antoinette Stettler, Kirchenfeldstrasse 45, 3005 Bern

Prof. Dr. Rudolf Trümpy, Allmendboden 19, 8700 Küsnacht

Prof.Dr. Brian Vickers, Chair of English Literature, Centre for Renaissance Studies, ETH-

Zentrum, Rämistrasse 101, 8092 Zürich

Prof. Dr. Hans H. Walser, Mühlebachstrasse 82, 8008 Zürich

Dr. Marcel Weber, Rudishaldenstrasse 8, 8800 Thalwil

Dr. habil. Karl Heinrich Wiederkehr, Birkenau 24, D-2000 Hamburg 76

Prof. Dr. Bernhard Zimmermann, Elisabeth-Mühlenweg-Strasse 13, D-78476 Allensbach

Prof. Dr. Heinrich Zollinger, Technisch-chemisches Laboratorium, ETH, 8092 Zürich 